\title{
Energy Scale Deformation on Regular Polyhedra
}

\author{
Takuya Eguchi ${ }^{1}$, Satoshi Oga ${ }^{2}$, Hosho Katsura $^{3,4,5}$, Andrej Gendiar ${ }^{6}$, and Tomotoshi Nishino ${ }^{1 *}$ \\ ${ }^{1}$ Department of Physics, Graduate School of Science, Kobe University, Kobe 657-8501, Japan \\ ${ }^{2}$ The Institute for Solid State Physics, The University of Tokyo, Kashiwa, Chiba 277-8581, Japan \\ ${ }^{3}$ Department of Physics, The University of Tokyo, 7-3-1 Hongo, Bunkyo-ku, Tokyo 113-0033, Japan \\ ${ }^{4}$ Institute for Physics of Intelligence, The University of Tokyo, 7-3-1 Hongo, Bunkyo-ku, Tokyo 113-0033, Japan \\ ${ }^{5}$ Trans-scale Quantum Science Institute, University of Tokyo, Bunkyo-ku, Tokyo 113-0033, Japan \\ ${ }^{6}$ Institute of Physics, Slovak Academy of Sciences, Bratislava 845 11, Slovakia
}

\begin{abstract}
A variant of energy scale deformation is considered for the $S=1 / 2$ antiferromagnetic Heisenberg model on polyhedra. The deformation is induced by the perturbations to the uniform Hamiltonian, whose coefficients are determined by the bond coordinates. On the tetrahedral, octahedral, and cubic clusters, the perturbative terms do not affect the ground state of the uniform Hamiltonian when they are sufficiently small. On the other hand, for the icosahedral and dodecahedral clusters, it is numerically confirmed that the ground state of the uniform Hamiltonian is almost insensitive to the perturbations unless they lead to a discontinuous change in the ground state. The obtained results suggest the existence of a generalization of sine-square deformation in higher dimensions.
\end{abstract}

\section{Introduction}

Uniformity in quantum states is one of the fundamental properties in condensed matter physics. On a regular lattice, the ground state of a quantum model is expected to be uniform when the Hamiltonian is translationally invariant unless spatial modulations are spontaneously stabilized. Occasionally, the ground state is uniform even when the Hamiltonian is not translationally invariant. For example, when the excitation gap is sufficiently large, the effect of slowly varying perturbations on the uniform ground state is negligible.

A class of non-uniform Hamiltonians, whose ground states are nearly uniform, is known in one dimension. Suppose that we have a one-dimensional lattice Hamiltonian

$$
\hat{H}=\sum_{\ell} \hat{h}_{\ell, \ell+1},
$$

where $\hat{h}_{\ell, \ell+1}$ represents the nearest-neighbor interaction, whose magnitude does not depend on the site index $\ell$. In the case of the translationally invariant quantum Heisenberg spin chain, $\hat{h}_{\ell, \ell+1}$ is written as the exchange interaction $J \hat{\boldsymbol{S}}_{\ell} \cdot \hat{\boldsymbol{S}}_{\ell+1}$, where $\hat{\boldsymbol{S}}_{\ell}$ denotes the spin operator at site $\ell$, and $J$ is the interaction parameter. In what follows, we assume that the ground state is uniform, and the excitation gap is zero in the thermodynamic limit. Introducing a deformation function $f_{\ell}$, which varies slowly with respect to $\ell$, we can modify the energy scale of each bond and define the non-uniform Hamiltonian

$$
\hat{H}_{f}=\sum_{\ell} f_{\ell} \hat{h}_{\ell, \ell+1} \text {. }
$$

When the function is exponential, i.e., $f_{\ell}=e^{\ell / \lambda},{ }^{1)}$ the groundstate of $\hat{H}_{f}$ is uniform in the bulk part of the system. ${ }^{2)}$ Under this exponential deformation, the correlation length becomes finite, and increases with the deformation parameter $\lambda>0$. A similar uniformity of the ground state has been observed for the hyperbolic deformation function $f_{\ell}=\cosh (\ell / \lambda){ }^{3-5)}$

The specific form of deformation that we focus on in this

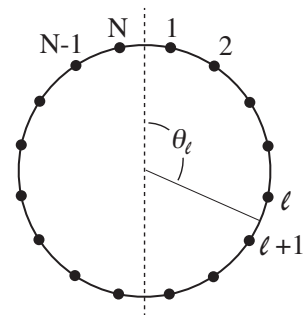

(a)

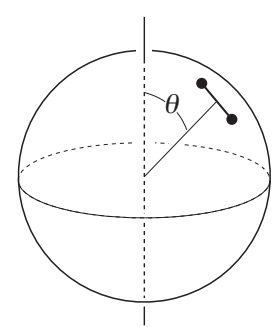

(b)
Fig. 1. (a) Angle $\theta_{\ell}$ contained in $\hat{H}_{\mathrm{M}}$ in Eq. (6). (b) Angle $\theta$ for a bond that connects lattice points on the sphere.

article is the sine-square deformation (SSD) ${ }^{6-8)}$ Consider the $\mathrm{N}$-site system whose Hamiltonian is written as

$$
\hat{H}_{\mathrm{SSD}}=\sum_{\ell=1}^{N} 2\left[\sin \frac{\ell \pi}{N}\right]^{2} \hat{h}_{\ell, \ell+1},
$$

where we have used the labeling rule that identifies $\ell=N+1$ with $\ell=1$. The prefactor of $\hat{h}_{N, 1}$ is zero, and therefore there is no coupling between the ends $\ell=1$ and $\ell=N$. Thus, the system can be considered as the finite-size system of length $N$ with open and smooth boundary conditions, ${ }^{9,10)}$ where the interaction strength decreases toward the both ends of the system. It was accidentally found that the ground state is uniform under the SSD when the free fermionic lattice model is considered. ${ }^{6,7)}$ In the correlated systems, the uniformity under the SSD was numerically confirmed for the Kondo lattice model, ${ }^{11)}$ the $S=1 / 2$ Heisenberg spin chain, ${ }^{8)}$ and the Hubbard model. ${ }^{12)}$ Theoretical proof of complete uniformity is given for the free fermionic lattice model. ${ }^{13,14)}$ It has been known that the continuum limit of the SSD has a natural interpretation in terms of conformal field theory (CFT). ${ }^{15-41)}$ Generalizations of the SSD to two dimensions were considered on finite lattices with torus, ${ }^{42)}$ disk,${ }^{43-45)}$ and tube geometries. ${ }^{44,46)}$ 
The deformation function in Eq. (3) can be written as

$$
f_{\ell}=2\left[\sin \frac{\ell \pi}{N}\right]^{2}=1-\cos \frac{2 \ell \pi}{N} .
$$

Accordingly, we can decompose $\hat{H}_{\mathrm{SSD}}$ into a sum of the uniform part

$$
\hat{H}_{0}=\sum_{\ell=1}^{N} \hat{h}_{\ell, \ell+1}
$$

and the modulated part

$$
\hat{H}_{\mathrm{M}}=-\sum_{\ell=1}^{N} \cos \frac{2 \ell \pi}{N} \hat{h}_{\ell, \ell+1} .
$$

A simple geometrical interpretation is possible for the angle $\theta_{\ell}=2 \ell \pi / N$ in Eq. (6), as shown in Fig. 1(a). When the lattice sites are located equidistantly on the circle, $\theta_{\ell}$ corresponds to the angle between the midpoint of the bond $(N, 1)$ and that of the bond $(\ell, \ell+1)$ measured from the center of the circle. In the case of the free-fermion hopping model on the lattice, it is straightforward to show that the ground state $\left|\psi_{0}\right\rangle$ of the uniform part $\hat{H}_{0}$ is an eigenstate of the modulated part $\hat{H}_{\mathrm{M}}$ with eigenvalue zero. It has been analytically shown that the generalized Hamiltonian

$$
\hat{H}(\gamma)=\hat{H}_{0}+\gamma \hat{H}_{\mathrm{M}}
$$

shares the same ground state $\left|\psi_{0}\right\rangle$ within the range $|\gamma| \leq$ $1 .^{13,42)}$

From the construction of $\hat{H}_{\mathrm{M}}$ in Eq. (6), which is related to the $N$-sided regular polygon in Fig. 1(a), it is possible to state that $\hat{H}_{\mathrm{M}}$ corresponds to the most slowly varying sinusoidally modulated function on the finite lattice. This geometric observation suggests a new type of two-dimensional generalization of the SSD. Let us imagine discrete lattices drawn on a sphere. The possible candidates are finite lattices in the shape of regular polyhedra, which are tetrahedron $(N=4)$, octahedron $(N=6)$, cube $(N=8)$, icosahedron $(N=12)$, and dodecahedron $(N=20)$. Ground-state properties of the $S=1 / 2$ antiferromagnetic Heisenberg model on these lattices have been known for the uniform case $\hat{H}_{0}^{(N)}{ }^{47-54)} \mathrm{Re}-$ call that the most slowly varying function on the unit sphere is the spherical harmonic function $Y_{1}^{0} \propto \cos \theta$, where $\theta$ represents the angle from a fixed axis, and $\cos \theta$ represents the coordinate component along the axis. Figure 1(b) shows the angle $\theta$ for a bond that connects lattice points on the sphere. We thus introduce the modulated part $\hat{H}_{M}^{(N)}$ that is the sum of the non-uniform nearest-neighbor interactions whose coefficients are specified by a linear function of coordinates of each bond. In this article, we examine the effect of $\hat{H}_{M}^{(N)}$ by means of obtaining the ground state of the combined Hamiltonian $\hat{H}^{(N)}=\hat{H}_{0}^{(N)}+c \hat{H}_{M}^{(N)}$. For $N=4,6$, and 8 , it is confirmed that the ground state of $\hat{H}_{0}^{(N)}$ is also the eigenstate of $\hat{H}_{M}^{(N)}$ with eigenvalue zero, and thus the ground state of $\hat{H}^{(N)}$ is independent of $c$ when $|c|$ is relatively small. For $N=12$ and 20 , the ground state of $\hat{H}_{M}^{(N)}$ depends on $c$, but the observed $c$-dependences are very weak.

The structure of this article is as follows. In the next section, we consider the tetrahedral cluster, which can be treated analytically. In Sect. 3, the octahedral and cubic clusters are examined. In Sect. 4 , the icosahedral and dodecahedral clus-
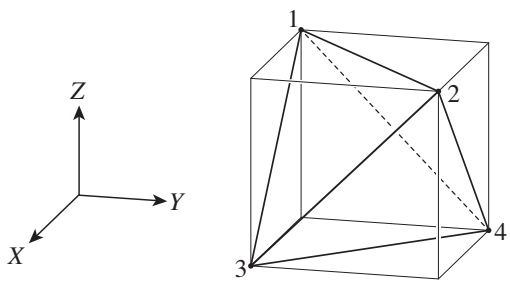

Fig. 2. Positions of sites on the tetrahedral cluster.

ters are examined. In these cases, the modulation changes the ground state, but the effect is very weak. Conclusions are summarized in the last section. We discuss possible generalizations of the SSD in higher dimensions.

\section{Energy Scale Deformation on the Tetrahedral Cluster}

Consider the $S=1 / 2$ antiferromagnetic Heisenberg model on finite lattices in the shape of regular polyhedra. Throughout this article, we assume only the nearest-neighbor interactions. We set the interaction parameter $J$ to unity, and thus the interaction between the neighboring sites $i$ and $j$ is simply expressed as $\hat{h}_{i, j}=\hat{\boldsymbol{S}}_{i} \cdot \hat{\boldsymbol{S}}_{j}$.

To become familiar with polyhedral geometries, we start with the tetrahedral cluster shown in Fig. 2, which is drawn inside the cube. The coordinates of the $\ell$-th site $\ell:(x, y, z)$ are fixed to

$$
\begin{aligned}
& 1:(-1,-1,1), \quad 2:(1,1,1), \\
& 3:(1,-1,-1), \quad 4:(-1,1,-1),
\end{aligned}
$$

where we set the origin at the center of the cube. The uniform Hamiltonian on the cluster is represented as

$$
\begin{aligned}
\hat{H}_{0}^{(4)} & =\hat{\boldsymbol{S}}_{2} \cdot \hat{\boldsymbol{S}}_{3}+\hat{\boldsymbol{S}}_{1} \cdot \hat{\boldsymbol{S}}_{4}+\hat{\boldsymbol{S}}_{2} \cdot \hat{\boldsymbol{S}}_{4}+\hat{\boldsymbol{S}}_{1} \cdot \hat{\boldsymbol{S}}_{3}+\hat{\boldsymbol{S}}_{1} \cdot \hat{\boldsymbol{S}}_{2}+\hat{\boldsymbol{S}}_{3} \cdot \hat{\boldsymbol{S}}_{4} \\
& =\frac{1}{2}\left(\hat{\boldsymbol{S}}_{1}+\hat{\boldsymbol{S}}_{2}+\hat{\boldsymbol{S}}_{3}+\hat{\boldsymbol{S}}_{4}\right)^{2}-\frac{3}{2} .
\end{aligned}
$$

We have explicitly shown the number of sites $N=4$ in the notation $\hat{H}_{0}^{(4)}$. This is an example of the Majumdar-Ghosh lattice. ${ }^{55)}$ Equation (9) clearly shows that the model $\hat{H}_{0}^{(4)}$ has two degenerate ground states with total spin 0 , each of which can be written as a product of singlet pairs. The other eigenstates of $\hat{H}_{0}^{(4)}$ have a nonzero total spin.

In order to introduce spatial modulations to the interactions, we focus on the coordinate of the midpoint of each bond. For example, they are $(0,0,1)$ and $(0,0,-1)$, respectively, for the bonds $(1,2)$ and $(3,4)$. If we use the $Z$-component of the midpoint coordinate as the prefactor to the corresponding pairwise interaction, we obtain the following modulated part:

$$
\hat{H}_{Z}^{(4)}=\hat{S}_{1} \cdot \hat{S}_{2}-\hat{S}_{3} \cdot \hat{S}_{4} \text {. }
$$

It can be easily verified that $\hat{H}_{Z}^{(4)}$ commutes with $\hat{H}_{0}^{(4)}$. Therefore, the combined Hamiltonian

$$
\begin{aligned}
\hat{H}^{(4)}(\gamma)= & \hat{H}_{0}^{(4)}+\gamma \hat{H}_{Z}^{(4)} \\
= & \hat{\boldsymbol{S}}_{2} \cdot \hat{\boldsymbol{S}}_{3}+\hat{\boldsymbol{S}}_{1} \cdot \hat{\boldsymbol{S}}_{4}+\hat{\boldsymbol{S}}_{2} \cdot \hat{\boldsymbol{S}}_{4}+\hat{\boldsymbol{S}}_{1} \cdot \hat{\boldsymbol{S}}_{3} \\
& +(1+\gamma) \hat{\boldsymbol{S}}_{1} \cdot \hat{\boldsymbol{S}}_{2}+(1-\gamma) \hat{\boldsymbol{S}}_{3} \cdot \hat{\boldsymbol{S}}_{4}
\end{aligned}
$$

shares the same ground state within the range $|\gamma|<1$, and the ground-state crossover occurs at $\gamma= \pm 1$. 

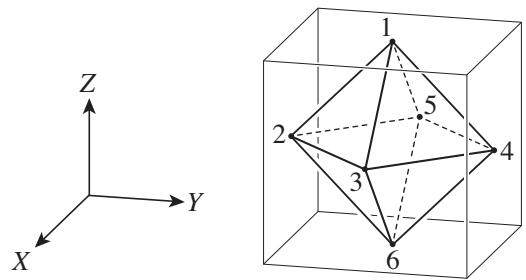

Fig. 3. Positions of sites on the octahedral cluster.

and the $Y$-components to obtain different types of modulated parts

$$
\begin{aligned}
& \hat{H}_{X}^{(4)}=\hat{\boldsymbol{S}}_{2} \cdot \hat{\boldsymbol{S}}_{3}-\hat{\boldsymbol{S}}_{1} \cdot \hat{\boldsymbol{S}}_{4}, \\
& \hat{H}_{Y}^{(4)}=\hat{\boldsymbol{S}}_{2} \cdot \hat{\boldsymbol{S}}_{4}-\hat{\boldsymbol{S}}_{1} \cdot \hat{\boldsymbol{S}}_{3} .
\end{aligned}
$$

A simple analysis shows that the ground state of the combined Hamiltonian

$$
\hat{H}^{(4)}(\alpha, \beta, \gamma)=\hat{H}_{0}^{(4)}+\alpha \hat{H}_{X}^{(4)}+\beta \hat{H}_{Y}^{(4)}+\gamma \hat{H}_{Z}^{(4)}
$$

is independent of the coefficients $\alpha, \beta$, and $\gamma$ within the range ${ }^{56)}$

$$
\alpha^{2}+\beta^{2}+\gamma^{2} \leq 3 \text { and } \alpha^{2}+\beta^{2}+\gamma^{2}+2 \alpha \beta \gamma \leq 1 .
$$

Typical values of the parameters at the boundary of the above region are $(\alpha, \beta, \gamma)=\left(\frac{1}{\sqrt{2}}, \frac{1}{\sqrt{2}}, 0\right),\left(\frac{1}{2}, \frac{1}{2}, \frac{1}{2}\right)$, and $(-1,-1,-1)$.

The analysis of the tetrahedral cluster has shown that the perturbative terms $\hat{H}_{X}^{(4)}, \hat{H}_{Y}^{(4)}$, and $\hat{H}_{Z}^{(4)}$ do not alter the ground state of $\hat{H}_{0}^{(4)}$ if their magnitudes are sufficiently small. In the following sections, we will observe similar results for the ground states of larger polyhedral clusters.

\section{On the Octahedral and Cubic Clusters}

The second system we consider is the octahedral cluster shown in Fig. 3, where the site coordinates are fixed as

$$
\begin{aligned}
& 1:(0,0,1), \quad 2:(0,-1,0), 3:(1,0,0) \\
& 4:(0,1,0), 5:(-1,0,0), 6:(0,0,-1) \text {. }
\end{aligned}
$$

In this case, the uniform Hamiltonian is given by

$$
\begin{aligned}
\hat{H}_{0}^{(6)} & =\hat{\boldsymbol{S}}_{1} \cdot \hat{\boldsymbol{S}}_{2}+\hat{\boldsymbol{S}}_{1} \cdot \hat{\boldsymbol{S}}_{3}+\hat{\boldsymbol{S}}_{1} \cdot \hat{\boldsymbol{S}}_{4}+\hat{\boldsymbol{S}}_{1} \cdot \hat{\boldsymbol{S}}_{5} \\
& +\hat{\boldsymbol{S}}_{2} \cdot \hat{\boldsymbol{S}}_{3}+\hat{\boldsymbol{S}}_{3} \cdot \hat{\boldsymbol{S}}_{4}+\hat{\boldsymbol{S}}_{4} \cdot \hat{\boldsymbol{S}}_{5}+\hat{\boldsymbol{S}}_{5} \cdot \hat{\boldsymbol{S}}_{2} \\
& +\hat{\boldsymbol{S}}_{2} \cdot \hat{\boldsymbol{S}}_{6}+\hat{\boldsymbol{S}}_{3} \cdot \hat{\boldsymbol{S}}_{6}+\hat{\boldsymbol{S}}_{4} \cdot \hat{\boldsymbol{S}}_{6}+\hat{\boldsymbol{S}}_{5} \cdot \hat{\boldsymbol{S}}_{6},
\end{aligned}
$$

which has a non-degenerate ground state $\left|\psi_{0}^{(6)}\right\rangle$. On the lattice, the $Z$-components of the midpoints of the bonds are $1 / 2$ for $(1,2),(1,3),(1,4)$, and $(1,5)$, and are $-1 / 2$ for $(2,6),(3,6)$, $(4,6)$, and $(5,6)$, and are 0 otherwise. To simplify the notation, we multiply the factor 2 to these $Z$-components to define the modulated part

$$
\begin{aligned}
\hat{H}_{Z}^{(6)} & =\hat{\boldsymbol{S}}_{1} \cdot \hat{\boldsymbol{S}}_{2}+\hat{\boldsymbol{S}}_{1} \cdot \hat{\boldsymbol{S}}_{3}+\hat{\boldsymbol{S}}_{1} \cdot \hat{\boldsymbol{S}}_{4}+\hat{\boldsymbol{S}}_{1} \cdot \hat{\boldsymbol{S}}_{5} \\
& -\hat{\boldsymbol{S}}_{2} \cdot \hat{\boldsymbol{S}}_{6}-\hat{\boldsymbol{S}}_{3} \cdot \hat{\boldsymbol{S}}_{6}-\hat{\boldsymbol{S}}_{4} \cdot \hat{\boldsymbol{S}}_{6}-\hat{\boldsymbol{S}}_{5} \cdot \hat{\boldsymbol{S}}_{6} .
\end{aligned}
$$

Although $\hat{H}_{Z}^{(6)}$ does not commute with $\hat{H}_{0}^{(6)}$, the relation

$$
\hat{H}_{Z}^{(6)}\left|\psi_{0}^{(6)}\right\rangle=0
$$

holds, and thus $\left|\psi_{0}^{(6)}\right\rangle$ is an eigenstate of $\hat{H}_{Z}^{(6)}$ with eigenvalue

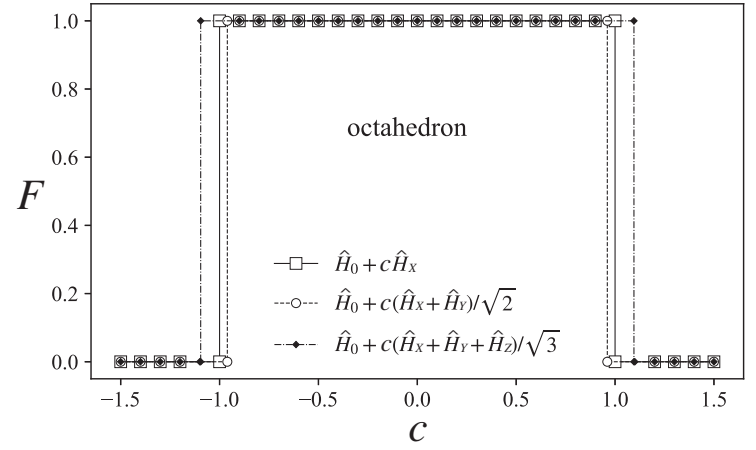

Fig. 4. Fidelity $F^{(6)}$ in the octahedral cluster.

use the $X$ - and $Y$-components of the coordinates, respectively, to define

$$
\begin{aligned}
\hat{H}_{X}^{(6)} & =\hat{\boldsymbol{S}}_{3} \cdot \hat{\boldsymbol{S}}_{1}+\hat{\boldsymbol{S}}_{3} \cdot \hat{\boldsymbol{S}}_{2}+\hat{\boldsymbol{S}}_{3} \cdot \hat{\boldsymbol{S}}_{6}+\hat{\boldsymbol{S}}_{3} \cdot \hat{\boldsymbol{S}}_{4} \\
& -\hat{\boldsymbol{S}}_{1} \cdot \hat{\boldsymbol{S}}_{5}-\hat{\boldsymbol{S}}_{2} \cdot \hat{\boldsymbol{S}}_{5}-\hat{\boldsymbol{S}}_{6} \cdot \hat{\boldsymbol{S}}_{5}-\hat{\boldsymbol{S}}_{4} \cdot \hat{\boldsymbol{S}}_{5}
\end{aligned}
$$

and

$$
\begin{aligned}
\hat{H}_{Y}^{(6)} & =\hat{\boldsymbol{S}}_{4} \cdot \hat{\boldsymbol{S}}_{1}+\hat{\boldsymbol{S}}_{4} \cdot \hat{\boldsymbol{S}}_{3}+\hat{\boldsymbol{S}}_{4} \cdot \hat{\boldsymbol{S}}_{6}+\hat{\boldsymbol{S}}_{4} \cdot \hat{\boldsymbol{S}}_{5} \\
& -\hat{\boldsymbol{S}}_{1} \cdot \hat{\boldsymbol{S}}_{2}-\hat{\boldsymbol{S}}_{3} \cdot \hat{\boldsymbol{S}}_{2}-\hat{\boldsymbol{S}}_{6} \cdot \hat{\boldsymbol{S}}_{2}-\hat{\boldsymbol{S}}_{5} \cdot \hat{\boldsymbol{S}}_{2},
\end{aligned}
$$

where the relations $\hat{H}_{X}^{(6)}\left|\psi_{0}^{(6)}\right\rangle=0$ and $\hat{H}_{Y}^{(6)}\left|\psi_{0}^{(6)}\right\rangle=0$ are also satisfied.

Analogous to Eq. (14), we introduce the linear combination

$$
\hat{H}^{(6)}(\alpha, \beta, \gamma)=\hat{H}_{0}^{(6)}+\alpha \hat{H}_{X}^{(6)}+\beta \hat{H}_{Y}^{(6)}+\gamma \hat{H}_{Z}^{(6)},
$$

and regard it as the deformed Hamiltonian. Note that $\left|\psi_{0}^{(6)}\right\rangle$ is an eigenvector of $\hat{H}^{(6)}(\alpha, \beta, \gamma)$. In order to determine the parameter region where $\left|\psi_{0}^{(6)}\right\rangle$ is the ground state of $\hat{H}^{(6)}(\alpha, \beta, \gamma)$, we numerically diagonalize $\hat{H}^{(6)}(\alpha, \beta, \gamma)$ to obtain its ground state $\left|\varphi^{(6)}(\alpha, \beta, \gamma)\right\rangle$, and calculate the fidelity

$$
\begin{aligned}
F^{(6)}(\alpha, \beta, \gamma) & =\left|\left\langle\varphi^{(6)}(\alpha, \beta, \gamma) \mid \psi_{0}^{(6)}\right\rangle\right| \\
& =\left|\left\langle\varphi^{(6)}(\alpha, \beta, \gamma) \mid \varphi^{(6)}(0,0,0)\right\rangle\right| .
\end{aligned}
$$

Throughout this article, we assume that all the states are normalized. We trace $F^{(6)}(\alpha, \beta, \gamma)$ typically along the paths on which the parameters are given by

$$
\begin{aligned}
& \text { (I) } \alpha=c, \quad \beta=0, \quad \gamma=0, \\
& \text { (II) } \alpha=\frac{c}{\sqrt{2}}, \quad \beta=\frac{c}{\sqrt{2}}, \quad \gamma=0, \\
& \text { (III) } \alpha=\frac{c}{\sqrt{3}}, \quad \beta=\frac{c}{\sqrt{3}}, \quad \gamma=\frac{c}{\sqrt{3}},
\end{aligned}
$$

where the factor $c$ denotes the magnitude of deformation. Figure 4 shows the calculated result. The fidelity $F^{(6)}$ is equal to unity for small $|c|$, and jumps to zero at (I) $|c|=1$, (II) $|c|=0.9608$, and (III) $|c|=1.0954$, where we have used the parametrization in Eqs. (24)-(26). In cases (II) and (III), a pairwise interaction with negative coefficient appears in $\hat{H}^{(6)}(\alpha, \beta, \gamma)$ in the neighborhood of the jumping point.

The third system we consider is the cubic cluster shown in Fig. 5, where the uniform Hamiltonian is written as

$$
\hat{H}_{0}^{(8)}=\hat{\boldsymbol{S}}_{1} \cdot \hat{\boldsymbol{S}}_{2}+\hat{\boldsymbol{S}}_{2} \cdot \hat{\boldsymbol{S}}_{3}+\hat{\boldsymbol{S}}_{3} \cdot \hat{\boldsymbol{S}}_{4}+\hat{\boldsymbol{S}}_{4} \cdot \hat{\boldsymbol{S}}_{1}
$$




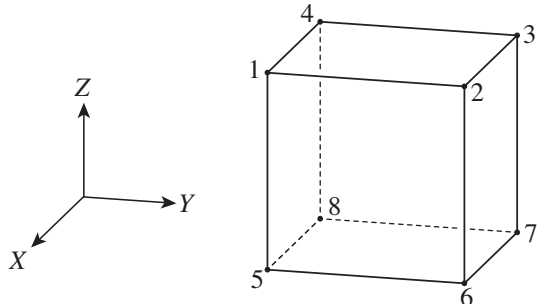

Fig. 5. Positions of sites on the cubic cluster.

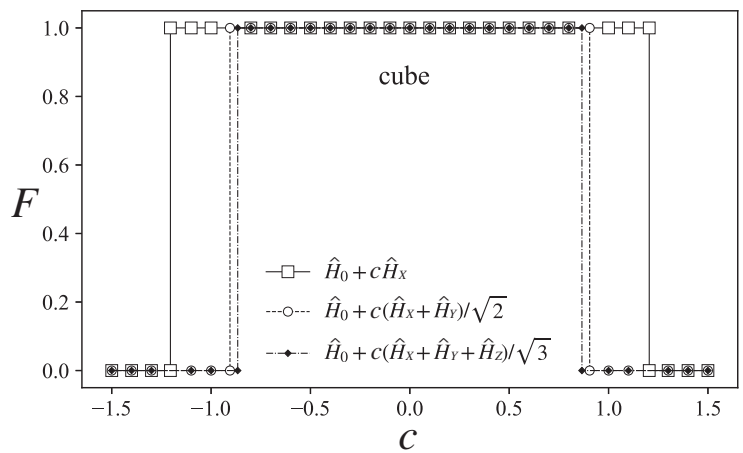

Fig. 6. Fidelity $F^{(8)}$ in the cubic cluster.

$$
\begin{aligned}
& +\hat{S}_{1} \cdot \hat{S}_{5}+\hat{\boldsymbol{S}}_{2} \cdot \hat{\boldsymbol{S}}_{6}+\hat{\boldsymbol{S}}_{3} \cdot \hat{\boldsymbol{S}}_{7}+\hat{\boldsymbol{S}}_{4} \cdot \hat{\boldsymbol{S}}_{8} \\
& +\hat{S}_{5} \cdot \hat{\boldsymbol{S}}_{6}+\hat{\boldsymbol{S}}_{6} \cdot \hat{\boldsymbol{S}}_{7}+\hat{\boldsymbol{S}}_{7} \cdot \hat{\boldsymbol{S}}_{8}+\hat{\boldsymbol{S}}_{8} \cdot \hat{\boldsymbol{S}}_{5},
\end{aligned}
$$

which has a non-degenerate ground state $\left|\psi_{0}^{(8)}\right\rangle$. In this case, it would be easy to capture the lattice geometry and obtain the coordinates of the midpoints of bonds. Similar to Eqs. (18), (20), and (21), the modulated parts are given by

$$
\begin{aligned}
\hat{H}_{X}^{(8)} & =\hat{\boldsymbol{S}}_{1} \cdot \hat{\boldsymbol{S}}_{2}+\hat{\boldsymbol{S}}_{2} \cdot \hat{\boldsymbol{S}}_{6}+\hat{\boldsymbol{S}}_{6} \cdot \hat{\boldsymbol{S}}_{5}+\hat{\boldsymbol{S}}_{5} \cdot \hat{\boldsymbol{S}}_{1} \\
& -\hat{\boldsymbol{S}}_{4} \cdot \hat{\boldsymbol{S}}_{3}-\hat{\boldsymbol{S}}_{3} \cdot \hat{\boldsymbol{S}}_{7}-\hat{\boldsymbol{S}}_{7} \cdot \hat{\boldsymbol{S}}_{8}-\hat{\boldsymbol{S}}_{8} \cdot \hat{\boldsymbol{S}}_{4}, \\
\hat{H}_{Y}^{(8)} & =\hat{\boldsymbol{S}}_{2} \cdot \hat{\boldsymbol{S}}_{3}+\hat{\boldsymbol{S}}_{3} \cdot \hat{\boldsymbol{S}}_{7}+\hat{\boldsymbol{S}}_{7} \cdot \hat{\boldsymbol{S}}_{6}+\hat{\boldsymbol{S}}_{6} \cdot \hat{\boldsymbol{S}}_{2} \\
& -\hat{\boldsymbol{S}}_{1} \cdot \hat{\boldsymbol{S}}_{4}-\hat{\boldsymbol{S}}_{4} \cdot \hat{\boldsymbol{S}}_{8}-\hat{\boldsymbol{S}}_{8} \cdot \hat{\boldsymbol{S}}_{5}-\hat{\boldsymbol{S}}_{5} \cdot \hat{\boldsymbol{S}}_{1},
\end{aligned}
$$

and

$$
\begin{aligned}
\hat{H}_{Z}^{(8)} & =\hat{\boldsymbol{S}}_{1} \cdot \hat{\boldsymbol{S}}_{2}+\hat{\boldsymbol{S}}_{2} \cdot \hat{\boldsymbol{S}}_{3}+\hat{\boldsymbol{S}}_{3} \cdot \hat{\boldsymbol{S}}_{4}+\hat{\boldsymbol{S}}_{4} \cdot \hat{\boldsymbol{S}}_{1} \\
& -\hat{\boldsymbol{S}}_{5} \cdot \hat{\boldsymbol{S}}_{6}-\hat{\boldsymbol{S}}_{6} \cdot \hat{\boldsymbol{S}}_{7}-\hat{\boldsymbol{S}}_{7} \cdot \hat{\boldsymbol{S}}_{8}-\hat{\boldsymbol{S}}_{8} \cdot \hat{\boldsymbol{S}}_{5} .
\end{aligned}
$$

Although the modulated parts $\hat{H}_{X}^{(8)}, \hat{H}_{Y}^{(8)}$, and $\hat{H}_{Z}^{(8)}$ do not commute with $\hat{H}_{0}^{(8)}$, the relations

$$
\hat{H}_{X}^{(8)}\left|\psi_{0}^{(8)}\right\rangle=0, \quad \hat{H}_{Y}^{(8)}\left|\psi_{0}^{(8)}\right\rangle=0, \quad \text { and } \hat{H}_{Z}^{(8)}\left|\psi_{0}^{(8)}\right\rangle=0
$$

are satisfied.

As was done in Eq. (22), we consider the combined Hamiltonian $\hat{H}^{(8)}(\alpha, \beta, \gamma)=\hat{H}_{0}^{(8)}+\alpha \hat{H}_{X}^{(8)}+\beta \hat{H}_{Y}^{(8)}+\gamma \hat{H}_{Z}^{(8)}$. Note that $\left|\psi_{0}^{(8)}\right\rangle$ is an eigenvector of $\hat{H}^{(8)}(\alpha, \beta, \gamma)$. In order to determine the parameter region where $\left|\psi_{0}^{(8)}\right\rangle$ is the ground state, we numerically diagonalize $\hat{H}^{(8)}(\alpha, \beta, \gamma)$ and obtain the corresponding ground state $\left|\varphi^{(8)}(\alpha, \beta, \gamma)\right\rangle$. Figure 6 shows the fidelity $F^{(8)}(\alpha, \beta, \gamma)=\left|\left\langle\varphi^{(8)}(\alpha, \beta, \gamma) \mid \psi_{0}^{(8)}\right\rangle\right|$. Under the parametrization in Eqs. (24)-(26), the fidelity $F^{(8)}$ is equal to unity
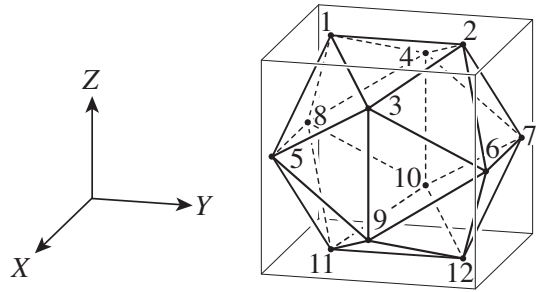

Fig. 7. Positions of sites on the icosahedral cluster.

$|c|=0.9050$, and (III) $|c|=0.8660$, where the ground state alternates. In cases (I) and (II), a pairwise interaction with negative coefficient appears in $\hat{H}^{(8)}(\alpha, \beta, \gamma)$ in the neighborhood of the jumping point.

On the tetrahedral $(N=4)$, octahedral $(N=6)$, and cubic $(N=8)$ clusters, we have confirmed that the ground state of the uniform part $\hat{H}_{0}^{(N)}$ is also a zero-energy eigenstate of the modulated parts $\hat{H}_{X}^{(N)}, \hat{H}_{Y}^{(N)}$, and $\hat{H}_{Z}^{(N)}$. This is the reason why the fidelity $F^{(N)}$ is unity in these systems, when the magnitude of modulation $|c|$ is relatively small.

\section{On the Icosahedral and Dodecahedral Clusters}

Let us move on to the icosahedral cluster shown in Fig. 7. To save space, we will not write down the explicit form of the uniform part $\hat{H}_{0}^{(12)}$, which is nothing but the sum of Heisenberg interactions $\hat{\boldsymbol{S}}_{i} \cdot \hat{\boldsymbol{S}}_{j}$ between neighboring sites. As we have considered in the previous sections, the modulated part is defined through the coordinates of the midpoints of the bonds. The golden ratio

$$
\phi=\frac{1+\sqrt{5}}{2}
$$

plays an important role in writing the coordinates simply. From the $Z$-component of the midpoint of each bond, we obtain the modulated part

$$
\begin{aligned}
H_{Z}^{(12)} & =S_{1} \cdot S_{2} \\
& +\frac{\phi}{2}\left(S_{1} \cdot S_{3}+S_{2} \cdot S_{3}+S_{2} \cdot S_{4}+S_{1} \cdot S_{4}\right) \\
& +\frac{1}{2}\left(S_{1} \cdot S_{5}+S_{1} \cdot S_{8}+S_{2} \cdot S_{6}+S_{2} \cdot S_{7}\right) \\
& +\frac{1}{2 \phi}\left(S_{3} \cdot S_{5}+S_{3} \cdot S_{6}+S_{4} \cdot S_{7}+S_{4} \cdot S_{8}\right) \\
& -\frac{1}{2 \phi}\left(S_{9} \cdot S_{5}+S_{9} \cdot S_{6}+S_{10} \cdot S_{7}+S_{10} \cdot S_{8}\right) \\
& -\frac{1}{2}\left(S_{11} \cdot S_{5}+S_{11} \cdot S_{8}+S_{12} \cdot S_{6}+S_{12} \cdot S_{7}\right) \\
& -\frac{\phi}{2}\left(S_{11} \cdot S_{9}+S_{12} \cdot S_{9}+S_{12} \cdot S_{10}+S_{11} \cdot S_{10}\right) \\
& -S_{11} \cdot S_{12} \cdot
\end{aligned}
$$

In the same manner, we can write down $\hat{H}_{X}^{(12)}$ and $\hat{H}_{Y}^{(12)}$, respectively, using the $X$ - and $Y$-components. Note that $\hat{H}_{X}^{(12)}$ and $\hat{H}_{Y}^{(12)}$ can be written just by replacing the lattice indices in Eq. (33) appropriately. In this case, the ground state of $\hat{H}^{(12)}$ is not an eigenstate of $\hat{H}_{X}^{(12)}, \hat{H}_{Y}^{(12)}$, nor $\hat{H}_{Z}^{(12)}$.

As we have done in the previous section, we consider the 


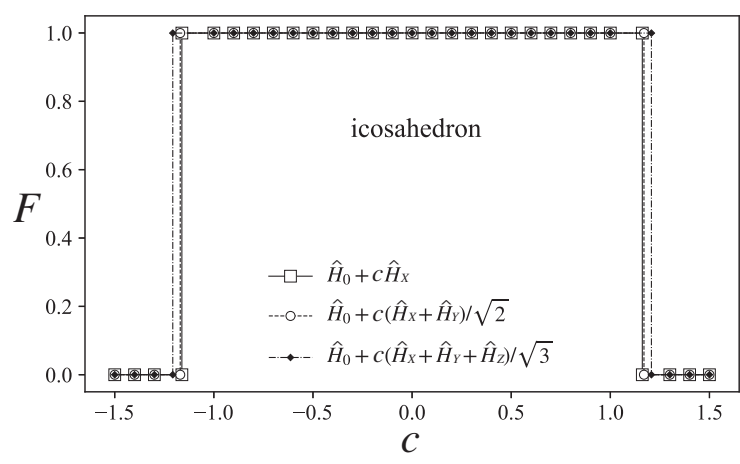

Fig. 8. Fidelity $F^{(12)}$ in the icosahedral cluster.
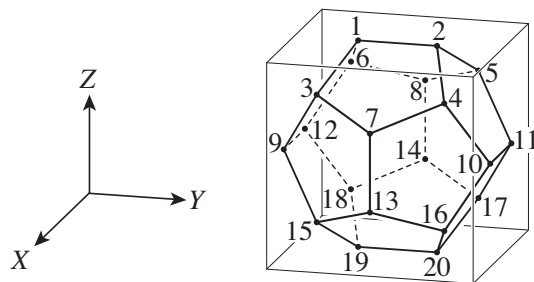

Fig. 9. Positions of sites on the dodecahedral cluster.

combined Hamiltonian $\hat{H}^{(12)}=\hat{H}_{0}^{(12)}+\alpha \hat{H}_{X}^{(12)}+\beta \hat{H}_{Y}^{(12)}+$ $\gamma \hat{H}_{Z}^{(12)}$, and obtain the ground state $\left|\varphi^{(12)}(\alpha, \beta, \gamma)\right\rangle$ by means of numerical diagonalization. Figure 8 shows the fidelity $F^{(12)}(\alpha, \beta, \gamma)=\left|\left\langle\varphi^{(12)}(\alpha, \beta, \gamma) \mid \varphi^{(12)}(0,0,0)\right\rangle\right|$. Under the parametrization in Eqs. (24)-(26), $F^{(12)}$ is close to unity when $|c|$ is small, and slightly decreases with $|c|$. The values of $c$ and $F^{(12)}$ at the border where the fidelity changes discontinuously are (I) $|c|=1.1613$ and $F^{(12)}=0.9997$, (II) $|c|=1.1697$ and $F^{(12)}=0.9997$, and (III) $|c|=1.2072$ and $F^{(12)}=0.9996$. In all the cases, some of the pairwise interactions in $\hat{H}^{(12)}(\alpha, \beta, \gamma)$ have a negative coefficient in the neighborhood of the border.

The last example we consider is the dodecahedral cluster shown in Fig. 9. The uniform part $\hat{H}_{0}^{(20)}$ is the sum of neighboring Heisenberg interactions. The modulated part $H_{Z}^{(20)}$ is given by

$$
\begin{aligned}
H_{Z}^{(20)} & =S_{1} \cdot S_{2} \\
& +\frac{\phi}{2}\left(S_{1} \cdot S_{3}+S_{2} \cdot S_{4}+S_{2} \cdot S_{5}+S_{1} \cdot S_{6}\right) \\
& +\frac{1}{2}\left(S_{3} \cdot S_{7}+S_{7} \cdot S_{4}+S_{5} \cdot S_{8}+S_{8} \cdot S_{6}\right) \\
& +\frac{1}{2 \phi}\left(S_{3} \cdot S_{9}+S_{4} \cdot S_{10}+S_{5} \cdot S_{11}+S_{6} \cdot S_{12}\right) \\
& -\frac{1}{2 \phi}\left(S_{9} \cdot S_{15}+S_{10} \cdot S_{16}+S_{11} \cdot S_{17}+S_{12} \cdot S_{18}\right) \\
& -\frac{1}{2}\left(S_{15} \cdot S_{13}+S_{13} \cdot S_{16}+S_{17} \cdot S_{14}+S_{14} \cdot S_{18}\right) \\
& -\frac{\phi}{2}\left(S_{15} \cdot S_{19}+S_{16} \cdot S_{20}+S_{17} \cdot S_{20}+S_{18} \cdot S_{19}\right) \\
& -S_{19} \cdot S_{20},
\end{aligned}
$$

where $\hat{H}_{X}^{(20)}$ and $\hat{H}_{Y}^{(20)}$ can be written in the same manner. Also

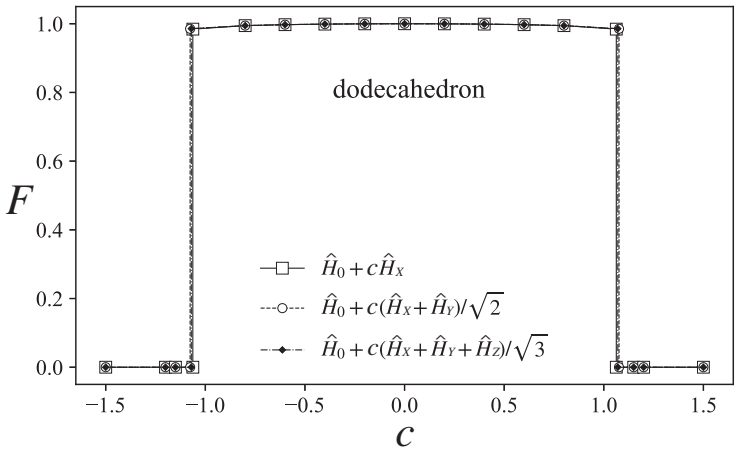

Fig. 10. Fidelity $F^{(20)}$ in the dodecahedral cluster.

in this case, the ground state of $\hat{H}^{(20)}$ is not an eigenstate of $\hat{H}_{X}^{(20)}, \hat{H}_{Y}^{(20)}$, nor $\hat{H}_{Z}^{(20)}$.

We consider the combined Hamiltonian $\hat{H}_{0}^{(20)}+$ $\alpha \hat{H}_{X}^{(20)}+\beta \hat{H}_{Y}^{(20)}+\gamma \hat{H}_{Z}^{(20)}$, and obtain the ground state $\left|\varphi^{(20)}(\alpha, \beta, \gamma)\right\rangle$ by means of the numerical Lanczos diagonalization. Figure 10 shows the fidelity $F^{(20)}(\alpha, \beta, \gamma)=\left|\left\langle\varphi^{(20)}(\alpha, \beta, \gamma) \mid \varphi^{(20)}(0,0,0)\right\rangle\right|$. Under the parametrization in Eqs. (24)-(26), $F^{(20)}$ is close to unity when $|c|$ is small, and slightly decreases with $|c|$. The values of $c$ and $F^{(20)}$ at the border where the fidelity changes discontinuously are (I) $|c|=1.0628$ and $F^{(20)}=0.9843$, (II) $|c|=0.9843$ and $F^{(20)}=0.9853$, and (III) $|c|=1.07047$ and $F^{(20)}=0.9857$. In case (I), one of the pairwise interactions in $\hat{H}^{(20)}(\alpha, \beta, \gamma)$ has a negative coefficient near the border.

\section{Conclusion and Discussion}

We have examined the effect of energy scale deformation applied to the antiferromagnetic Heisenberg model on the polyhedral clusters. The deformation is introduced by the perturbative Hamiltonian, which is defined through the coordinate of the midpoint of each bond. In the tetrahedral, octahedral, and cubic clusters, the ground states are not modified at all by the energy scale deformation, up to a certain amount of deformation magnitude. In the icosahedral and dodecahedral clusters, the ground state is slightly modified, but the effect of perturbation is very weak.

In our trial of the energy scale deformation, we used linear functions of the midpoint coordinate of each bond as the prefactor of the modulated part. There would be a better construction of the modulated part also in the icosahedral and dodecahedral clusters, where the uniform Hamiltonian and the modulated part share a common eigenstate. Since the parameter space of the prefactors is finite, one way to clarify this issue is to perform a parameter search numerically. We expect that the symmetries of the polyhedra restrict the number of independent parameters, thereby making the analysis simpler. Another possible approach is to find out the most slowly varying function on the polyhedral lattice by means of the diagonalization of the one-particle hopping Hamiltonian on the lattice. Note that the generation of an orthonormal set by diagonalization can be generalized to finite lattices with planar geometry, such as square lattices with rectangular or disk geometry, ${ }^{43-45)}$ with appropriate boundary conditions. The nearly uniform and the most slowly varying functions, respectively, 
The deformation effect can also be examined on the Archimedean solids, the quasi-regular polyhedra with a larger number of sites ${ }^{50,54,57-64)}$ such as the C60 "buckyball", 47, 65, 66) An interesting question to ask is whether the deformation effect decreases with the number of sites $N$ towards the continuous limit on the sphere. In four dimensions, there are several regular polytope (or poly-cell) models, and the effect of energy scale deformation can be considered on these systems. For the largest case, the 600-cell $(N=600)$, one must employ the tensor network method to obtain the ground state.

The authors are grateful to K. Okunishi for valuable discussions. T. N. acknowledges support from JSPS KAKENHI Grant Nos. 17K05578 and 21K03403. H. K. was supported by JSPS Grant-in-Aid for Scientific Research on Innovative Areas No. JP20H04630, JSPS KAKENHI Grant No. JP18K03445, and the Inamori Foundation.

1) K. Okunishi, J. Phys. Soc. Jpn. 76, 063001 (2007).

2) K. Okunishi and T. Nishino, Phys. Rev. B 82, 144409 (2010).

3) H. Ueda and T. Nishino, J. Phys. Soc. Jpn. 78, 014001 (2009).

4) H. Ueda, H. Nakano, K. Kusakabe, and T. Nishino, Prog. Theor. Phys. 124, 389 (2010)

5) H. Ueda, H. Nakano, K. Kusakabe, and T. Nishino, J. Phys. Soc. Jpn. 80, 094001 (2011).

6) A. Gendiar, R. Krcmar, and T. Nishino, Prog. Theor. Phys. 122, 953 (2009).

7) A. Gendiar, R. Krcmar, and T. Nishino, Prog. Theor. Phys. 123, 393 (2010).

8) T. Hikihara and T. Nishino, Phys. Rev. B 83, 060414(R) (2011).

9) M. Vekić and S. R. White, Phys. Rev. Lett. 71, 4283 (1993)

10) M. Vekić and S. R. White, Phys. Rev. B 53, 14552 (1996).

11) N. Shibata and C. Hotta, Phys. Rev. B 84, 115116 (2011).

12) A. Gendiar, M. Daniska, Y. Lee, and T. Nishino, Phys. Rev. A 83, 052118 (2011).

13) H. Katsura, J. Phys. A: Math. Theor. 44, 252001 (2011).

14) K. Okunishi and H. Katsura, J. Phys. A: Math. Theor. 48, 445208 (2015).

15) H. Katsura, J. Phys. A: Math Theor. 45, 115003 (2012).

16) T. Tada, Mod. Phys. Lett. A 30, 1550092 (2015).

17) N. Ishibashi and T. Tada, J. Phys. A: Math. Theor. 48, 315402 (2015).

18) N. Ishibashi and T. Tada, Int. J. Mod. Phys. A 31, 1650170 (2016).

19) K. Okunishi, Prog. Theor. Exp. Phys. 2016, 063 A02 (2016).

20) X. Wen, S. Ryu, and A. W. W. Ludwig, Phys. Rev. B 93, 235119 (2016)

21) S. Tamura and H. Katsura, Prog. Theor. Exp. Phys. 2017, 113A01 (2017).

22) T. Tada, Prog. Theor. Exp. Phys. 2018, 061 B01 (2018).

23) X. Wen and J. Q. Wu, Phys. Rev. B 97, 184309 (2018).

24) X. Wen and J. Q. Wu, arXiv:1805.00031.

25) I. Kishimoto, T. Kitade, and T. Takahashi, Prog. Theor. Exp. Phys. 2018 123B04 (2018).

26) S. Zeze, Turk J. Phys. 43, 393 (2019).

27) I. MacCormack, A. Liu, M. Nozaki, and S. Ryu, J. Phys. A: Math. Theor. 52, 505401 (2019).
28) T. Tada, J. Phys. A: Math. Theor. 53, 255401 (2020).

29) T. Mendes-Santos, G. Giudici, M. Dalmonte, and M. A. Rajabpour, Phys. Rev. B 100, 155122 (2019).

30) R. Fan, Y. Gu, A. Vishwanath, and X. Wen, Phys. Rev. X 10, 031036 (2020).

31) C. Chen, arXiv:1908.10777.

32) B. Lapierre, K. Choo, C. Tauber, A. Tiwari, T. Neupert, and R. Chitra, Phys. Rev. Res. 2, 023085 (2020).

33) X. Liu and T. Tada, Prog. Theor. Exp. Phys. 2020, 061 B01 (2020).

34) P. Caputa, and I. MacCormack, and J. High Energy Phys. 2021, 27 (2021).

35) B. Lapierre, K. Choo, A. Tiwari, C. Tauber, T. Neupert, and R. Chitra Phys. Rev. Res. 2, 033461 (2020)

36) X. Wen, R. Fan, A. Vishwanath, and Y. Gu, Phys. Rev. Res. 3, 023044

(2021).

37) D. S. Ageev, A. A. Bagrov, and A. A. Iliasov, Phys. Rev. B 103, 100302 (2021).

38) B. Han and X. Wen, Phys. Rev. B 102, 205125 (2020).

39) M. Andersen, F. Nørfjand, and N. T. Zinner, Phys. Rev. D 103, 056005 (2021).

40) R. Fan, Y. Gu, A. Vishwanath, and X. Wen, SciPost Phys. 10, 049 (2021).

41) D. Das, R. Ghosh, and K. Sengupta, J. High Energy Phys. 2021, 172 (2021).

42) I. Maruyama, H. Katsura, and T. Hikihara, Phys. Rev. B 84, 165132 (2011).

43) C. Hotta, S. Nishimoto, and N. Shibata, Phys. Rev. B 87, 115128 (2013)

44) S. Nishimoto, N. Shibata, and C. Hotta, Nat. Commun. 4, 2287 (2013).

45) C. Hotta and K. Asano, Phys. Rev. B 98, 140405 (2018).

46) K. Yonaga and N. Shibata, J. Phys. Soc. Jpn. 84, 094706 (2015).

47) N. A. Modine and E. Kaxiras, Phys. Rev. B 53, 2546 (1996).

48) N. P. Konstantinidis, Phys. Rev. B 72, 064453 (2005).

49) N. P. Konstantinidis, Phys. Rev. B 76, 104434 (2007).

50) N. P. Konstantinidis, Phys. Rev. B 80, 134427 (2009).

51) N. P. Konstantinidis, J. Phys. Condens. Matter 27, 076001 (2015).

52) K. Karlova, J. Strecka, and J. Richter, J. Phys.: Condens. Matter 29, 125802 (2017).

53) N. P. Konstantinidis, J. Phys.: Condens. Matter 33, 325801 (2021).

54) S. G. Tabrizi and C. A. Jiménez-Hoyos, arxiv:2109.08041.

55) C. K. Majumdar and D. Ghosh, J. Math. Phys. 10, 1388 (1969).

56) Interestingly, the second relation in Eq. (15) appears in the following article: M. Kohmoto, B. Sutherland, and C. Tang, Phys. Rev. B 35, 1020 (1987).

57) D. Coffey, and S. A. Trugman, Phys. Rev. B 46, 12717 (1992).

58) J. Schnack, H. J. Schmidt, J. Richter, and J. Schulenburg, Eur. Phys. J. B 24, 475 (2001).

59) M. Exler and J. Schnack, Phys. Rev. B 67, 094440 (2003).

60) J. Schnack, J. Low. Temp. Phys. 142, 279 (2006)

61) I. Rousochatzakis, A. M. Läuchli, and F. Mila, Phys. Rev. B 77, 094420 (2008).

62) J. Schnack, Dalton Trans. 39, 4677 (2010).

63) J. Ummethuma and J. Schnack, Polyhedron 66, 28 (2013).

64) J. Ummethuma, J. Schnack, and A. M. Läuchli, J. Magn. Magn. Mater. 327, 103 (2013).

65) D. Coffey and S. A. Trugman, Phys. Rev. Lett. 69, 176 (1992).

66) R. Rausch, C. Plorin, and M. Peschke, arXiv:2011.12083. 